Meta

Journal des traducteurs

Translators' Journal

\title{
La traductologie, l'ethnographie et la production des connaissances
}

\section{Hélène Buzelin}

Volume 49, numéro 4, décembre 2004

URI : https://id.erudit.org/iderudit/009778ar

DOI : https://doi.org/10.7202/009778ar

Aller au sommaire du numéro

\section{Éditeur(s)}

Les Presses de l'Université de Montréal

ISSN

0026-0452 (imprimé)

1492-1421 (numérique)

Découvrir la revue

Citer cet article

Buzelin, H. (2004). La traductologie, l'ethnographie et la production des connaissances. Meta, 49(4), 729-746. https://doi.org/10.7202/009778ar

\section{Résumé de l'article}

La traductologie s'est récemment enrichie d'un nouveau courant de recherche d'orientation postcoloniale. En ce qu'il remet en question les notions d'équivalence et de transfert sur lesquelles se fondaient les théories traditionnelles, ce courant jette les bases d'un questionnement épistémologique analogue à celui auquel les ethnographes ont procédé, il y a une quarantaine d'années. L'analogie entre la figure de l'ethnographe et celle du traducteur, leur rôle en tant qu'interprètes culturels et écrivains, a fait l'objet de plusieurs analyses. Cet article propose de creuser l'analogie en la resituant toutefois en amont. Il explore en quoi la réflexion des ethnographes sur leurs propres pratiques d'interprétation et sur la production des connaissances peut éclairer, aujourd'hui, celle des traductologues. L'hypothèse qui le sous-tend est la suivante : la perspective ethnographique, en particulier les travaux de Bruno Latour, nous invite à penser la traduction comme un processus de production qui fait appel à des intermédiaires opérant selon une logique de réseau. Dans ce cadre, la traduction-texte n'apparaît plus comme le " simple » reflet des normes d'une société donnée ou de la subjectivité d'un traducteur, mais bien plutôt comme l'expression des relations qui se sont tissées entre ces intermédiaires. Cette conclusion permet de valider les modèles traductologiques fondés sur les notions de dialectisme et d'hybridité, tout en évitant le biais textuel et littéraire qui les caractérise. Ce faisant, elle entraîne un élargissement du débat éthique et ouvre de nouvelles avenues de recherche pour le paradigme descriptif.
Ce document est protégé par la loi sur le droit d'auteur. L’utilisation des services d'Érudit (y compris la reproduction) est assujettie à sa politique d'utilisation que vous pouvez consulter en ligne.

https://apropos.erudit.org/fr/usagers/politique-dutilisation/ 


\title{
La traductologie, l'ethnographie et la production des connaissances*
}

\author{
HÉLÈNE BUZELIN \\ Université de Montréal, Montréal, Canada \\ helene.buzelin@umontreal.ca
}

\section{RÉSUMÉ}

La traductologie s'est récemment enrichie d'un nouveau courant de recherche d'orientation postcoloniale. En ce qu'il remet en question les notions d'équivalence et de transfert sur lesquelles se fondaient les théories traditionnelles, ce courant jette les bases d'un questionnement épistémologique analogue à celui auquel les ethnographes ont procédé, il y a une quarantaine d'années. L'analogie entre la figure de l'ethnographe et celle du traducteur, leur rôle en tant qu'interprètes culturels et écrivains, a fait l'objet de plusieurs analyses. Cet article propose de creuser l'analogie en la resituant toutefois en amont. II explore en quoi la réflexion des ethnographes sur leurs propres pratiques d'interprétation et sur la production des connaissances peut éclairer, aujourd'hui, celle des traductologues. L'hypothèse qui le sous-tend est la suivante: la perspective ethnographique, en particulier les travaux de Bruno Latour, nous invite à penser la traduction comme un processus de production qui fait appel à des intermédiaires opérant selon une logique de réseau. Dans ce cadre, la traduction-texte n'apparaît plus comme le «simple» reflet des normes d'une société donnée ou de la subjectivité d'un traducteur, mais bien plutôt comme l'expression des relations qui se sont tissées entre ces intermédiaires. Cette conclusion permet de valider les modèles traductologiques fondés sur les notions de dialectisme et d'hybridité, tout en évitant le biais textuel et littéraire qui les caractérise. Ce faisant, elle entraîne un élargissement du débat éthique et ouvre de nouvelles avenues de recherche pour le paradigme descriptif.

\section{ABSTRACT}

The field of translation studies has only recently opened itself to the postcolonial. Inasmuch as postcolonial translation theories question the notions of equivalence and transfer on which traditional theories of translation were founded, they initiate an epistemological debate resembling that which marked the field of anthropology about forty years ago. A number of studies have explored the analogy between the role of translators and ethnographers as cultural interpreters and writers. This article carries on the investigation by exploring how the reflection of ethnographers on their own (and others') production of knowledge can enlighten that of contemporary translation theorists. The underlying hypothesis is the following: the ethnographic perspective, Bruno Latour's work in particular, invites us to conceive translation as a production process that relies on intermediaries operating in networks. Hence, the translated text does no longer appear as the "simple" reflection of the norms of a given society or that of the subjectivity of the translator, but rather as the expression of the relations between the various intermediaries that have participated in its production. This conclusion validates theoretical models based on notions of dialectism and hybridity while avoiding the textual and literary bias that have so far characterized them. By doing so, it calls for a widening of the ethical debate and opens new research avenues in descriptive translation studies.

\section{MOTS-CLÉS/KEYWORDS}

ethnographie, production des connaissances, traductologie, dialectisme, hybridité 


\begin{abstract}
To enter into the postcolonial world is to see cultural relations at a global level, to understand the complexities of the histories and power relations which operate across continents. For translation studies and literary study in general, adopting a postcolonial frame means enlarging the map which has traditionally bound literary and cultural studies. [...] And so 'we' must understand our own place on this map. Where do 'we' belong, where are 'we' speaking from, and on the basis of what particular kinds of knowledge? (Simon and St-Pierre 2000: 13-14).
\end{abstract}

Si la traductologie s'est constituée dans les années soixante-dix, en même temps que les études postcoloniales, elle a trouvé ses premiers fondements dans les théories linguistiques et culturelles européennes (du moins selon Jacquemond 1992) et, d'après certains (Lewis 2002; Cronin 2003), fait encore preuve de peu d'ouverture aux langues hybrides et minoritaires. Les premières études portant sur la traduction en contexte postcolonial sont effectivement apparues il y a une dizaine d'années à peine, et c'est au cours des cinq dernières, grâce (entre autres) aux travaux de Lawrence Venuti, Sherry Simon, Paul St-Pierre, Maria Tymozcko et Harish Trivedi, que s'est développé, au sein de la discipline, un courant de recherche à proprement parler. Or, en ce qu'il remet en question les modèles traditionnels fondés sur les notions de transfert et d'équivalence, en ce qu'il appelle les chercheurs à "prendre position » et à interroger les fondements de leurs discours et de leurs pratiques, ce courant jette les bases d'un questionnement analogue à celui qui a marqué, dans les années cinquante et soixante, le champ de l'anthropologie, questionnement dont les théoriciens de la traduction (qui tentaient alors de faire reconnaître la légitimité de leur objet d'étude et de créer un nouveau champ de recherche) avaient jusque-là fait l'économie. En quoi - sous quelles conditions et dans quelle mesure - la réflexion des anthropologues peut-elle bien éclairer celle qui s'esquisse, aujourd'hui, en traductologie?

Dès lors que l'on appréhende la culture comme un «texte» et que l'on voit dans les textes des représentations culturelles - ce à quoi conduit le "virage culturel» (Bassnett 1998) des études en traduction -, les liens entre l'ethnographie et la traductologie se resserrent d'un coup, les études des uns acquièrent une pertinence immédiate pour les autres. Depuis les quarante dernières années, les analogies ne cessent de se multiplier. Filant la métaphore de Clifford Geertz qui «lisait» la culture comme un texte et définissait l'ethnographe comme «traducteur culturel», certains anthropologues tels que Clifford et Marcus $(1986$; 1997) ont trouvé dans Walter Benjamin un maître à penser. À leur tour, les traductologues s'inspirent depuis peu des travaux de ces anthropologues pour étudier le lien entre la traduction interlinguistique, la littérature du voyage et l'écriture ethnographique (Valero-Garcès 1995; Tymoczko 1999; Cronin 2000; Wolf 2000; Polezzi 2001). Si l'ethnographie s'apparente à la traduction - et inversement -, les deux pratiques se sont traditionnellement distinguées sur au moins un aspect fondamental: littéraire ou pragmatique, la seconde n'est pas une discipline universitaire à proprement parler et n'a aucune prétention scientifique. Cette différence se répercute sur le plan de la recherche. Contrairement à l'anthropologie, la traductologie est une discipline où théorie et pratique ne sont pas indissociables et ont d'ailleurs été longtemps maintenues à distance. Pourtant, dès 1984, Berman voyait dans le clivage entre théoriciens et praticiens l'un des principaux obstacles des 
études en traduction. Afin d'éviter cet écueil, il proposait alors de définir la traductologie comme une réflexion sur la traduction «à partir de sa nature même d'expérience» (1984: 300). Son appel n'a pas été suivi, du moins pas immédiatement. Les traductologues, qu'ils travaillent dans une optique historiographique, sociologique ou philosophique, se sont avant tout penchés sur des textes «sacrés» (littéraires, religieux ou scientifiques) publiés et traduits par d'autres. Les chercheurs sont toutefois de plus en plus nombreux à souligner la nécessité de favoriser le rapprochement entre les sphères de la théorie et de la pratique. Pour Simeoni, la réhabilitation en traductologie du point de vue de l'agent serait la première condition d'un tel rapprochement: "short of taking the view of the translator in the act of translating as a solid reference point, any approach (and even more so, any theory) of translation or cultural transfer will run the risk of becoming estranged from its object. [...] The principled stance is one way of building into the field of translation studies a minimal condition for shared approaches to translating» (Simeoni 1995: 448). Cette optique converge avec celle de Cronin qui se prononce en faveur d'une pratique réflexive de la traduction: translation as reflexion, rather than reflection (Cronin 2003). Cette quête de réflexivité se manifeste plus particulièrement dans le domaine littéraire. Ces dernières années ont ainsi vu la parution de plusieurs études qui, par la réalisation de projets (re)-traduction d'œuvres romanesques, interrogent sur un mode inductif et réflexif, la nature du processus de traduction (Levine 1991, Kadish and MassardierKenney (eds.) 1994, Chapdelaine et Lane-Mercier (eds.) 2001, Buzelin 2004). C'est dans ce cadre, à partir du moment où pratique et théorie se rejoignent, que le parallèle entre les deux figures, celle de l'ethnographe et celle du traductologue (et non pas uniquement du traducteur) devient possible, que la réflexion des premiers peut enrichir celle des seconds, et ce à un niveau autre que métaphorique. Dans cet article, je m'intéresserai donc à la seconde partie de la question: quelle est la nature de cet apport?

À partir du $\mathrm{xx}^{\mathrm{e}}$ siècle, le terrain est devenu la pierre angulaire de la démarche anthropologique. Aussi, lorsque les anthropologues ont commencé à questionner leurs pratiques, ils ont interrogé, en premier lieu, leur rôle sur le terrain. Pour Mary Douglas, l'anthropologie (du moins l'anthropologie sociale britannique) des années cinquante se distinguait de par son intérêt pour l'épistémologie: «What was called 'field methods' was in fact learning to question the conditions of knowing» (1995: 24). De fait, cette décennie et la suivante virent la publication de plusieurs articles et monographies interrogeant les liens entre l'ethnographie et le colonialisme (Leiris 1950, Gough 1968, Leclerc 1972), les fondements épistémologiques de la discipline (Leach 1961, Maquet 1964, Wolf 1964, Berreman 1968, Scholte 1971, Hymes (ed.) 1972), les méthodes et relations sur le terrain (Jongmans et al. 1967, Nash and Wintrob 1972), l'éthique de la profession (Jarvie 1969, Jorgensen 1971, Fabian 1971). S’inspirant des théories littéraires et de la philosophie, les anthropologues de la génération suivante ont étudié les modes de représentation, les pratiques d'écriture ethnographique (Asad (ed.) 1973, Clifford 1980, Clifford et Marcus 1986, Said 1989, Pàlsson 1993). D’autres, tels que Bruno Latour, se sont tournés vers les processus d'innovation scientifique et technique, élargissant ainsi la réflexion sur la construction des connaissances au-delà de l'anthropologie. Par rapport aux anthropologues, les traductologues interrogent avant tout des représentations écrites. Aussi, les travaux anthropologiques qui ont aujourd'hui quelque écho en traductologie sont ceux qui 
questionnent les stratégies d'écriture ethnographique, en particulier ceux de Talal Asad (1973, 1979), de Clifford et Marcus (1986) et de James Clifford (1997). Pourtant, envisagées dans une perspective réflexive, les pratiques traductive et ethnographique ne se recoupent pas uniquement sur le plan de l'écriture. Si les traducteurs n'effectuent pas de "terrain", ils se documentent, se renseignent, consultent des sources, effectuent des recherches. En ce qu'elle nous éloigne du paradigme littéraire et textuel qui, selon Sela-Sheffy (2000), continue de grever les études en traduction, et nous invite plutôt à penser la traduction comme un processus de production, la réflexion des anthropologues permet d'aborder les notions de «sujet traduisant» sous un angle nouveau. Comme on le verra au fil de cet article, elle nous invite à reconnaître, et donc à étudier, la nature collective et dialogique de ce processus. Elle confirme ainsi les conclusions des sociologues quant à la nécessité d'étudier ce processus de l'intérieur, du point de vue de l'agent (Simeoni 1995). Mais elle nous permet aussi de les pousser plus loin: de voir dans la confrontation des points de vue entre les différents intermédiaires qui y sont engagés, un élément constitutif du processus en question. Cette perspective favorise un élargissement du débat éthique et ouvre de nouvelles avenues de recherche pour le courant descriptif.

\section{Empowering Translation (Studies)}

Pour saisir en quoi la réflexion anthropologique peut être enrichissante pour la traductologie, il faut commencer par rappeler les principales questions, les constats et options formulés par les traductologues travaillant dans une optique postcoloniale. Robinson (1997) ayant déjà effectué une synthèse de ce courant de recherche, je partirai de ses conclusions, en tenant compte des travaux publiés depuis les cinq dernières années. De façon générale, les recherches postcoloniales ont en commun d'envisager la traduction dans une perspective politique. La question qui sous-tend ces recherches pourrait se résumer ainsi: "Who is transforming what how? And also: if a current or still-dominant cultural transformation is harmful to our interests, how can we retranslate its terms so as to engineer a different transformation » (Robinson 1997: 93).

Alors que les tout premiers textes commentés par Robinson (1997), ceux de Rafael, de Cheyftiz et de Niranjana, ont surtout interrogé les pratiques de traduction durant l'époque coloniale, ceux qui ont été publiés depuis les cinq dernières années accordent une importance grandissante aux pratiques contemporaines. Analysant les flux de traduction (littéraire) au niveau mondial, Venuti (1998), Heilbron (1999) et Cronin (2003) ont confirmé, entre autres, l'asymétrie des échanges nord-sud relevée par Jacquemond (1992). Un courant de recherche, incarné par Samia Mehrez dans l'étude de Robinson, et qui s'est développé depuis, consiste à étudier les textes des écrivains postcoloniaux contemporains. Sherry Simon (1999) et Maria Tymoczko (1999b, 2000) creusent ainsi le parallèle entre les pratiques de ces écrivains postcoloniaux et la traduction interlinguistique ${ }^{1}$. Ces travaux cherchent moins à plaquer les concepts traductologiques à l'étude de textes en question, qu'à explorer en quoi ces derniers, de par leur hybridité, remettent en question les oppositions entre original et traduction. Qu'il s'agisse de l'Inde, la Caraïbe, l'Irlande, ou l'Afrique du Nord, les textes ou contextes contemporains étudiés par ces chercheurs ont en commun d'être des lieux marqués par le bilinguisme, la diglossie ou le multilinguisme, des lieux de 
friction linguistique et de traduction. Si l'histoire de la traduction en Europe est intimement liée aux processus de normalisation linguistique et au développement des États-nations (Lewis 2002), les modèles développés dans ce cadre s'appliquent difficilement aux contextes étudiés par ces chercheurs. Ici, la traduction n'apparaît plus comme une opération de transfert entre deux langues-cultures opposées et étrangères l'une à l'autre, mais comme un processus de transformation (Lewis 2002), une pratique de métissage (Nouss et Laplantine 1997) et d'hybridité (Wolf 2000), une zone de tension (Simon 1996) un instrument de construction d'identités collectives. Ainsi, les tendances à «diaboliser» la traduction relevées par Robinson (1997: 104106) s'estompent également. Les études de cas qui figurent dans les collectifs plus récents (Bassnett et Trivedi 1999, Simon et St-Pierre 2000) tendent plutôt à souligner le potentiel créatif et constructif de la traduction. En ce qu'elles réinscrivent leur objet dans son contexte (social, politique et historique), les recherches traductologiques d'orientation postcoloniale se situent dans le prolongement du paradigme descriptif envisagé par Holmes et développé par Toury (1980). Cela dit, dans la mesure où elles s'intéressent plus particulièrement au rôle de la traduction dans les luttes de pouvoir, démontrant en quoi cette pratique peut être tantôt dangereuse, émancipatrice, constructive ou aliénante, elles en modifient également l'agenda. Plus qu'une fenêtre (sur une société, une époque, un polysystème, une culture), la traduction dans une perspective postcoloniale devient un instrument. La description puis la reconnaissance des pouvoirs, dangers et limites de la traduction, débouche inévitablement sur une réflexion où se mêlent des considérations d'ordre éthique et épistémologique.

Dans l'avant-dernier chapitre de Translation and Empire intitulé «Resistance, redirection and Retranslation», Robinson évoque différentes «options» envisagées par les chercheurs travaillant dans une perspective postcoloniale, options par lesquelles la traduction pourrait devenir une pratique émancipatrice contribuant au rééquilibrage des rapports de force: le littéralisme selon Niranjana, les mistranslations selon Rafael et le métissage selon Mehrez. De ces trois options, la deuxième a eu relativement peu d'écho, la troisième valorise les pratiques des écrivains postcoloniaux polyglottes qui, refusant d'opter pour l'une ou l'autre langue, pratiquent le métissage et écrivent «entre» plusieurs langues. La première rejoint l'éthique du foreignizing préconisée par Lawrence Venuti, la politique de la traduction de Spivak et le respect de la lettre d'Antoine Berman. L'éthique de ces quatre traductologues se recoupe dans un même rejet de l'idéal de "communication", rejet hérité de la philosophie de Walter Benjamin (1923). Il est intéressant de constater que mis à part la deuxième, ces options renvoient toutes à des stratégies d'écriture et consistent, plus souvent qu'autrement, à traduire de façon littérale, du moins originale. Le texte est l'aboutissement, la finalité et ce qui reste du processus de traduction, ce par quoi les lecteurs accéderont, de façon partielle et médiatisée, au texte de départ et à une parcelle de la ou des cultures dont il procède ou qui s'en réclament. Ne serait-ce que pour cette raison, les modes de représentation sont effectivement déterminants, en traduction comme en anthropologie d'ailleurs ${ }^{2}$. Si le foreignizing, par l'utilisation de minority elements (Venuti 1996) puisés dans le contexte «d'arrivée », a l'avantage de rendre plus visible le travail de transformation à l'œuvre dans les processus de traduction et de favoriser l'hétérogénéité dans ce contexte, il comporte plusieurs limites que Robinson et plusieurs autres (y compris Venuti lui-même) n'ont pas manqué de souligner. Cette stratégie serait un peu trop élitiste. Elle reposerait sur des modèles binaires difficilement tenables dès 
lors qu'on se situe dans un paradigme théorique d'hybridité et de métissage. Ses vertus politiques n'iraient pas de soi (dans certains cas, l'ethnocentrisme pourrait être subversif, tout dépend du contexte de traduction: qui traduit et pour qui l'on traduit, etc.). Enfin et surtout, répondant aux critères de la «haute» littérature contemporaine, cette stratégie ne se prête pas à tous types de textes. Or, la littérature, et à plus forte raison la «haute» littérature, représente une partie infime de ce qui se traduit aujourd'hui.

S'il remet en question les notions de transfert et d'équivalence, le courant traductologique postcolonial, sans doute parce qu'il est encore très récent, continue de refléter le biais littéraire des études en traduction. Venuti, Spivak et Niranjana font de la traduction un instrument politique, mais l'éthique qu'ils proposent renvoie avant tout à des représentations, des «styles» d'écriture et perpétue dans une large mesure le «Culte» du texte. Selon Sela-Sheffy, cette sacralisation continue de grever les théories littéraires les plus récentes et, plus spécifiquement, les discours (théoriques ou non) sur la traduction. Selon elle, ce n'est donc pas dans l'opposition entre approches essentialiste ou non essentialiste (depuis longtemps dépassée en sciences sociales), mais bien plutôt autour du clivage texte vs. processus que se divise le champ de la traductologie. Soulignant la primauté des approches axées sur l'étude des textes, elle en appelle à un renversement des orientations de recherche: «expand[ing] the focus and interest of Translation Studies from the products (the translated texts) as such, to the interdependencies and exchange relations between products, persons (producers and consumers), institutions, and the translation market» (Sela-Sheffy 2000:348). Cette réorientation se justifie sur le double plan éthique et théorique. Comme le souligne Michael Cronin, «Textual scrupulousness is only certain good. There must be an activist dimension to translation which involves an engagement with the cultural politics of society at national and international levels» (Cronin 2003: 134). De même, envisager la traduction comme un instrument de pouvoir, une zone de tension, de métissage et donc une pratique dont l'issue serait en grande partie imprévisible a des conséquences théoriques majeures du point de vue des études descriptives. Ceci semble en effet remettre en question la possibilité même de définir des «lois » et d'établir des modèles prédictifs. À ces deux questions, éthique et théorique, l'anthropologie offre, il me semble, quelques éléments de réponse.

\section{L'ethnographie et la production des connaissances}

Les propos de Michael Cronin rappellent ceux que lancèrent, dans les années soixante, certains anthropologues britanniques et américains militant en faveur d'une anthropologie plus «engagée» (Davies 1999). Aux États-Unis, ces protestations furent déclenchées par une série de scandales (le projet Camelot en 1964 et la Thailand Controversy en 1970) liés à l'utilisation de recherches anthropologiques (financées) par l'armée américaine et ayant légitimé l'intervention armée dans des pays du tiers-monde. Elles suscitèrent un débat sur la responsabilité de l'anthropologue et l'éthique de la profession. Ces controverses ne sont toutefois que l'expression la plus radicale et le prolongement d'une réflexion épistémologique qui s'était amorcée au sortir de la Seconde Guerre mondiale. Si les raisons de cette «révolution» sont diverses et complexes ${ }^{3}$, l'élément déterminant demeure le processus de décolonisation: «The dissolution of empires entails the collapse of structures of knowledge. When an 
organization disintegrates, the forms of knowledge that have been called forth by the effort to organize collapse too. The process is sad for some, joyful for others » (Douglas 1995: 16). Le démantèlement des empires obligeait l'anthropologie à se réinventer (Berreman 1969), du moins à se repenser (Leach 1961).

La reconnaissance des transformations sur les sociétés dites "primitives » entraînées par l'imposition du régime colonial, son effritement puis son abolition, allait non seulement ternir l'image de l'ethnographe, elle ébranlait la principale dichotomie sur laquelle s'était fondée la discipline (opposition entre les sociétés «modernes » et «primitives»), les homologies qui s'y rattachaient (ici/ailleurs, présent/passé) et le paradigme épistémologique (objectivant) qui les alimentaient. Devant l'ampleur des mutations s'opérant non seulement dans les sociétés soi-disant «traditionnelles » mais également dans celles qui les avaient colonisées, l'approche - fonctionnaliste ou structuraliste - qui consistait à étudier les sociétés comme des touts homogènes et harmonieux en dehors des rapports et influences extérieures, approche qui tendait à figer l'objet d'étude dans un passé idéalisé, perdait sa valeur heuristique. Dès les années cinquante, plusieurs, dont Edmund R. Leach, critiquèrent le «biais ethnocentrique » de l'anthropologie fonctionnaliste de Radcliffe-Brown et Malinowski, en appelant les chercheurs à ne plus se lancer « dans un développement avec des concepts chargés de valeurs qui préjugent de toute la question» (Leach 1968: 37). Plusieurs, dont Bastide, Balandier et Gluckman, commencèrent également à s'intéresser aux processus de transformation, à voir dans ces transformations et disruptions un élément constitutif des cultures, donc essentiel à leur compréhension ${ }^{4}$. En outre, les troubles sociaux et politiques, les problèmes économiques du tiers-monde rendaient l'idéalisation à laquelle tendaient les théories structuralistes d'autant évidente et critiquable. La nouvelle orientation se justifiait ainsi sur le double plan théorique et politique, invitant les chercheurs, sinon à "s'engager", du moins à aborder des questions contemporaines et pertinentes pour ces sociétés. Elle était également, et dans une large mesure, le résultat de nouveaux compromis. En effet, l'accession à l'indépendance des sociétés dans lesquelles les anthropologues avaient traditionnellement réalisé leur terrain allait modifier la marge de manœuvre et les conditions d'exercice des chercheurs. Pour la première fois, ces derniers devaient soumettre leurs projets aux nouveaux gouvernements et en recevoir l'approbation. Sur le terrain, les pratiques allaient prendre également un tour différent: " under postcolonial conditions the social and cultural anthropologist tended to be drawn into a social system of inquiry which included tribesmen with their own viewpoints, sensitivities, and sufficient power to affect the course of research or stop it altogether» (Nash and Wintrop 1972: 531). Les chercheurs durent considérer la possibilité d'être lus par des membres des sociétés qu'ils avaient étudiées, s'attendre à ce que leurs interprétations soient en partie contestées. Dès lors, il devenait essentiel de prendre en compte, avant, pendant et après le terrain, le point de vue et les interprétations de ceux qui, jusque-là, n'avaient pas été considérés comme des lecteurs et critiques potentiels, mais comme de "simples» informateurs. Ces remises en question et nouveaux compromis expliquent, en partie, pourquoi le terrain est devenu matière à réflexion, des réflexions qui pour la première fois ne relevaient plus de l'anecdote. La prise en compte des conditions institutionnelles (durée moyenne du séjour, la formation - ou le peu de formation - le précédant, les compétences linguistiques) a révélé l'ambiguïté des notions de participation et d'engagement, l'ambiguïté de la position du chercheur, enfin et surtout sa vulnérabilité 
en tant qu'interprète culturel et les limites de son entreprise. Ses aptitudes à comprendre et à interpréter les «autres", jusque-là tenues pour acquises, étaient désormais mises en doute.

La remise en question de l'opposition entre sociétés «traditionnelles» et «modernes» et le contexte politique dans lequel elle s'inscrit allaient donc modifier la définition même de l'objet d'étude et, par conséquent, la façon de l'appréhender. Le chercheur dut commencer par reconnaître sa propre influence sur la construction et la compréhension de ces cultures «turn the telescope the other way round [creating] observational reciprocity» (Douglas 1995: 24), se définir non seulement comme un observateur partial, mais un acteur. Ainsi, "the ethnographic enterprise is not a matter of what one person does in a situation but how two sides of an encounter arrive at a delicate workable definition of their meeting» (Crick 1982: 25). À l'image du scientifique «collectant» des données, s'est substituée celle d'un interlocuteur; le dialogue entre le chercheur et ses informateurs devenant le principe fondateur de la démarche de recherche. Bref, non seulement le chercheur ne peut prétendre à une pleine compréhension, mais sa compréhension - aussi partielle/partiale soit-elle n'est possible qu'avec l'aide de ceux et celles qui, en y participant, donnent sens aux manifestations culturelles/sociales qu'il se propose d'étudier. Dès lors, le terrain allait prendre son sens et sa valeur dans l'interaction entre le chercheur et ses informateurs ou collaborateurs (Fabian 1983). Il se définissait comme un processus inscrit dans une logique d'échange, de réciprocité (Lundberg 1968), voire d'exploitation mutuelle (Hatfield 1975), échange au fil duquel les différentes parties, ayant leur propre agenda, divulguent certaines informations, en dissimulent d'autres (Crick 1982: 26), renégocient constamment leur relation, ajustent leur position. Le rejet des dichotomies sur lesquelles s'était construite l'anthropologie fonctionnaliste, et la valorisation parallèle d'une épistémologie dialectique, fondant la connaissance sur un principe d'échange et de confrontation, ont entraîné un «rapatriement» de l'anthropologie dans les sociétés occidentales et fait émerger une nouvelle figure, celle de l'ethnographe « indigène».

Lorsque les anthropologues occidentaux sont retournés chez eux, rappelle Bruno Latour, ils se sont penchés en premier lieu sur ce qui ressemblait le plus «aux terrains qu'ils venaient de quitter: les arts et traditions populaires, la sorcellerie, les représentations symboliques, les campagnes, les marginaux de tous ordres, les ghettos» (Latour et Woolgar 1988: 16). Dans un second temps, ils se sont intéressés aux sphères de pouvoir: la science, la technologie. La sociologie des réseaux de Bruno Latour et le courant de recherche qui s'y rattache ${ }^{5}$ étudient les processus d'innovation scientifique et technique sur le terrain, la science en action (1989). Selon ce chercheur, la modernité reposait sur deux pratiques complémentaires: 1) production/ multiplication d'hybrides ou «quasi-objets»; 2) travail de purification masquant le processus de production de ces hybrides. Dès lors, le travail du chercheur consiste à rétablir la symétrie, à démasquer ce travail de "purification» ainsi que les processus de production de ces quasi-objets. Fondée sur le rejet des dichotomies cartésiennes du type nature/culture, sujet/objet, cognitif/contextuel, homme/machine ayant longtemps délimité les objets et méthodes de recherche, la théorie de Latour fait appel à deux concepts clés: celui de réseau et celui de traduction. Ici, le second concept renvoie à un processus de médiation, «d'interprétation» d'objectifs, s'exprimant dans les «langues» des différents intermédiaires engagés dans un projet/processus d'inno- 
vation - intermédiaires dont, à l'origine, les points de vue et intérêts ne sont pas nécessairement les mêmes. Il désigne, en somme, l'ensemble des stratégies qui permettent aux objectifs de se transformer et de se déplacer entre les intermédiaires, assurant la participation de ces derniers et la poursuite du projet. Selon Latour, «L'erreur des modernes sur eux-mêmes est assez facile à comprendre une fois que l'on rétablit la symétrie et que l'on prend en compte à la fois le travail de purification et le travail de traduction. Ils ont confondu les produits et les procédés » (Latour 1997 : 156). Ainsi, à partir d'une étude ethnographique, in situ, des processus d'innovation, Latour s'est attaché à démontrer à quel point «la science ne se produit pas de façon plus scientifique que la technique de manière technique, que l'organisation de manière organisée ou l'économie et manière économique» (Latour 1997 : 157). Or, il est raisonnable de suggérer, à titre d'hypothèse, qu'il en va de la traduction comme de la production de n'importe quel autre produit...

\section{Qui traduit?}

\section{Le dialogisme, du texte aux agents}

Toute saisie d'un objet par un sujet constitue un filtrage, c'est-à-dire une médiation par le sujet récepteur. Celui-ci plaque sur l'objet la grille de présupposés culturels, idéologiques, expérientiels, intellectuels qu'il s'est constituée au fil d'une existence et, à moins de se faire violence pour résister à la tentation de caser l'objet nouveau dans les structures du connu, à moins de faire table rase de ses préjugés, ce qui exige une véritable ascèse d'anthropologue, il finit par ne reconnaître que ce qu'il a appris au préalable à connaître (Folkart 1991: 310).

Qu'il ait affaire à un texte issu de sa culture ou bien à un texte étranger, le traducteur plaquera sa propre grille et ses présupposés, ne traduira du texte que ce qu'il a pu y reconnaître et donc ce qu'il connaissait déjà. Il nous donnera à lire son interprétation, une interprétation parmi d'autres... à moins qu'il ne se fasse anthropologue. Nida et Mounin ont vanté depuis longtemps les vertus de la démarche ethnographique. Le premier y voyait en effet «the most fruitful approach to the semantic problems of translation» (Nida 1945: 207) et, quelques années plus tard, le second faisait des ethnographes ceux qui fondèrent «les possibilités d'une vraie théorie et d'une vraie pratique scientifique de la traduction» (Mounin 1963: 241). Les théories de la traduction de ces chercheurs se situaient toutefois dans un cadre résolument structuraliste et, du moins pour Nida, colonialiste; un cadre empruntant des postulats que les anthropologues ont depuis longtemps remis en question. L'ethnographe ne prétend plus «collecter» des données, mais envisage son travail comme un processus de construction reposant sur une confrontation de points de vue. Cet échange commence concrètement sur le terrain et se prolonge, de façon plus métaphorique, au fil du processus d'écriture. Les théoriciens qui proposent de penser la traduction comme une pratique de métissage (Berman 1984, Nouss et Laplantine 1997) ou d'hybridité (Wolf 2000) partagent cette épistémologie dialectique. Cela dit, réinscrit dans le champ de la traductologie, le dialogisme prend un sens légèrement différent. Il ne renvoie plus qu'à la seconde partie du processus: le travail d'écriture. L'échange interpersonnel qui le sous-tendait (du moins en ethnographie) est évacué. Il ne s'agit plus d'un dialogue entre des personnes, mais d'un dialogue entre un lecteur et son 
texte, voire entre deux textes. Bref, il ne reste plus que des représentations, au mieux un seul agent. Aussi fragmentée soit cette représentation ou l'identité de l'agent, l'expérience ne relève plus du social, mais de l'intime. On reconnaît là encore l'influence des théories littéraires qui, tout en valorisant l'hybridité, contribuent à maintenir une vision romantique et très individuelle du processus de création ou de traduction, nous invite à voir la traduction comme une pratique d'écriture et de lecture, mais un acte qui ne relève en aucun cas de la recherche ou de la production. Si, en adoptant le principe d'anthropologie symétrique de Latour, on interroge non plus uniquement les produits (textes), mais les procédés (le processus de production), le dialogisme peut alors prendre un nouveau sens.

Tout d'abord, partant de l'objet d'étude des traductologues postcoloniaux, c'està-dire la littérature postcoloniale, il est à noter que les textes en question font parfois, voire souvent, l'objet de projets de traduction collectifs rassemblant des lecteurs provenant d'horizons divers et donc susceptibles de porter, sur le texte à traduire, des regards différents. Qu'ils soient ou non motivés par la complexité linguistique et culturelle des textes, ces projets instaurent d'emblée une rupture avec les modèles associant la traduction à un processus individuel et binaire allant de l'étranger vers le domestique. Certaines de ces collaborations sont officielles, lorsque les traductions sont cosignées. C'est entre autres le cas des traductions des nouvelles de la romancière jamaïcaine Olive Senior par le GERB ${ }^{6}$ (un groupe de recherche en traduction littéraire de l'Université de Bordeaux), du roman Texaco de Chamoiseau par Rose-Myriam Rejouis et Val Vinokurov, pour ne citer que quelques exemples. Elles sont parfois officieuses. Dans ce cas, le lecteur apercevra alors dans le paratexte, en post- ou préface, le nom de quelques-unes des personnes ayant contribué à la réalisation du produit fini. Les projets collectifs, post-face et préfaces constituant souvent, pour le donneur d'ouvrage, un coût de production supplémentaire, la dimension collective du processus demeure généralement officieuse, et donc peu reconnue, peu documentée et peu valorisée. Cette dimension collective, qu'elle soit officieuse ou non, n'est toutefois pas propre à la traduction des littératures postcoloniales, ni même à la traduction littéraire. Elle ressort d'ailleurs assez clairement des quelques analyses réflexives aujourd'hui disponibles, y compris celles qui portent sur des projets de traduction $a$ priori individuels.

Ces analyses (Levine 1991, Kadish et Massardier-Kenney (eds.) 1994, Chapdelaine et Lane-Mercier (eds.) 2001, Buzelin 2004) ont révélé, entre autres, le rôle constitutif du «conflit des subjectivités» (Lane-Mercier 2001), des négociations, tensions, mais aussi des rapports de complicité qui jalonnent le processus de traduction. Ce faisant, elles confirment à quel point les normes ne sont pas purement objectives et imposées, leur formation relevant d'une logique complexe qui, pour être comprise, doit être appréhendée du point de vue de l'agent, ou plus exactement des agents. Enfin, en ce qu'elles questionnent, sur un mode empirique, l'apparente linéarité et l'individualité du processus de traduction, ces études signalent, en creux, la difficulté voire l'impossibilité de réifier ce processus. Elles confirment ainsi les constats des théoriciens postcoloniaux quant au caractère imprévisible du processus en question. Ce faisant, elles révèlent aussi un écueil méthodologique rencontré par les travaux traductologiques contemporains d'orientation sociologique. Car, qu'ils adoptent le modèle du champ, du polysystème ou de la sociocritique, ces travaux ont en commun d'aborder le processus traductionnel de façon rétrospective. Ces travaux ont certes le mérite de 
mettre en lumière les multiples enjeux que soulève la traduction littéraire, voire les aléas et imprévus qui entourent la réception du produit fini (qu'il s'agisse d'un texte ou d'un corpus de textes étranger). Cela dit, fondés sur un travail de reconstruction $a$ posteriori portant pour l'essentiel sur l'étude des textes après publication, ils tendent, en même temps, à privilégier les facteurs (formels ou contextuels) conduisant à l'établissement d'explications logiques et fonctionnalistes, explications empruntant, pour reprendre l'expression de Latour (1989: 214), des «modèles de diffusion». Ils minimisent de ce fait d'autres aspects également constitutifs du processus traductionnel (donc essentiels à sa compréhension), mais qui ne s'affichent pas comme tels dans le produit fini et s'effacent avec le temps : les négociations entre les agents, les imprévus et les stratégies de persuasion (ou détournements de stratégies) permettant de gérer ces imprévus ainsi que d'assurer et de guider la participation (ou démission) des agents. En somme, ces travaux nous renseignent utilement sur le rôle de la traduction dans un contexte social et historique donné mais, comme le souligne Juliane House, ils ne permettent pas entièrement de comprendre «how and why a translation qua translation is as it is» (House 2001: 245). À ce titre les études réflexives évoquées plus haut comportent elles aussi une limite de taille. Le processus y étant conjointement réalisé et étudié par des universitaires, dans un cadre universitaire, ces études excluent de la réflexion des facteurs qui acquerraient une dimension centrale dans un contexte authentique, maison d'édition ou bureau de traduction: la gestion du risque, des contraintes (juridique, financière, temporelle) ainsi que des ressources (matérielles et humaines, autres que les traducteurs) mises en ouvre, autant de facteurs susceptibles d'exacerber ce «conflit des subjectivités». Les conflits, tensions et négociations, voire les controverses font partie du processus de traduction. Les anecdotes ne manquent pas. Selon Crick (1982), les rapports interpersonnels, les relations de confiance ou de complicité, mais également les tensions, voire les échecs du terrain ethnographique étaient aussi un intarissable sujet d'anecdotes, jusqu'à ce que l'on y reconnaisse une dimension constitutive du processus en question. Au vu des constats et de l'orientation théorique auxquels convient les traductologues postcoloniaux, il semblerait nécessaire d'aller au-delà de l'anecdote. Autrement dit, après avoir reconnu la dimension collective du processus traductionnel, il conviendrait d'étudier les interactions entre les intermédiaires ( $\mathrm{y}$ compris les machines) participant à ce processus, d'étudier la traduction non seulement du point de vue de l'agent (Simeoni 1995), mais plus exactement des agents ou, si l'on emprunte la terminologie latourienne, des actants.

L'idée selon laquelle on traduit seul relève du cliché. Non seulement les traducteurs utilisent, et ont toujours utilisé, des ressources exogènes humaines et matérielles (logiciels, livres, auteur, informateurs, lecteurs-réviseurs plus ou moins formels, voire d'autres traducteurs s'ils sous-traitent), mais le processus de transfert linguistique s'accompagne d'une multiplicité de décisions qui leur échappent, et se prolonge en aval, à diverses étapes, comme la révision du manuscrit ou le choix du titre, élément pesant d'un poids considérable mais sur lequel les traducteurs n'ont pas toujours droit de regard. L'équation entre le processus de traduction à la figure du traducteur, déjà passablement réductrice, devient franchement trompeuse dans un contexte de globalisation. Comme le rappelle Cronin (2003), la mondialisation et l'intensification des échanges économiques entraînent une compression des délais de traduction. Dès lors, les processus de traduction et de production tendent à se confondre; les frontières entre les différentes étapes du processus s'estompent; la répartition des 
tâches se redéfinit et cette redéfinition, qui suppose une forme de négociation, se joue sur le terrain. Qu'ils s'intéressent à la traduction littéraire ou pragmatique, les traductologues sont de plus en plus nombreux à souligner la polyvalence dont les traducteurs doivent aujourd'hui faire preuve. Selon Spivak, la traductrice des littératures «du tiers-monde» doit non seulement avoir une maîtrise active des langues de départ et d'arrivée. Elle doit également être assez au fait de la production littéraire dans cette langue pour être en mesure d'y porter un regard critique, avoir «a tough sense of the specific terrain of the original» (Spivak 1993: 405), être attentive aux différences avant de rechercher les points communs. Comme le souligne Sherry Simon, les critères imposés sont élevés, peut-être trop exigeants et idéalistes si l'on tient compte des conditions d'exercice des traducteurs professionnels. Ces critères deviennent plus exigeants encore lorsqu'ils s'appliquent à des textes écrits non pas dans une mais plusieurs langues. Ils sont en effet difficilement atteignables aussi longtemps qu'on traduit de l'étranger vers le familier, et aussi longtemps que l'on ramène le processus de traduction à une seule et même personne. Dans un tout autre domaine, celui de la traduction publicitaire, Séguinot (1995) évoque l'importance des aptitudes des traducteurs dans le maniement des nouvelles technologies, le marketing, les questions juridiques; Simeoni (1998: 31) souligne quant à lui «the sudden valuation of automated skills such as "product formating» in a profession that used to be mostly concerned with style». Selon ce chercheur, les changements qui affectent aujourd'hui la profession «point to increasing demands on a cognitive faculty that is only marginally present in linguistic or even more generally languagebased models, i.e. the adaptive faculty» (Simeoni 1998: 31). Dans la même veine, Defeng Li (2000) mentionne la nécessité de développer les aptitudes des étudiants à travailler en groupe.

Tandis que se redéfinissent les rôles, les aptitudes et les tâches (et les écoles de traduction refondent leurs curriculums) la relation entre le processus de transfert linguistique, la subjectivité du traducteur et le produit fini se complexifie. Non seulement le traducteur n'est pas le seul à traduire, et ne traduit pas seul, mais son rôle ne saurait se réduire au processus de transfert interlinguistique. En ce sens, le produit fini ne peut plus s'expliquer non plus dans des termes purement subjectifs (le traducteur) ou objectifs (le contexte historique, le polysystème). L'explication est à rechercher, plutôt, dans le processus même de fabrication de ce produit, dans la façon dont les ressources (humaines, technologiques, financières, etc.) mobilisées ont été mises en relation. Telle est la conclusion à laquelle conduit la reconnaissance, au-delà du texte, du caractère hybride, productif et dialogique du processus de traduction. L'intérêt de la réflexion anthropologique, en particulier celle de Bruno Latour, est de procurer un cadre théorique et méthodologique dans lequel l'étude empirique des processus de traduction, maintenant les partis pris d'hybridité et de métissage, demeure envisageable. La démarche de Latour consiste à suivre les processus de production in situ, et donc à étudier les pratiques de façon serrée, autant que possible en accompagnant les projets en cours. Adaptée à l'étude des processus de traduction, cette approche permettrait de rendre compte des «multiples mains» qui participent au processus et de générer ainsi des données auxquelles les traductologues ont rarement eu accès jusqu'ici'. Une telle approche permettrait d'étudier la façon dont ces intermédiaires (à commencer par le traducteur) interagissent, négocient leur position et leurs objectifs; la façon dont chacun inscrit sa participation et oriente la 
forme du produit fini. Elle permettrait d'étudier le rôle des alliances, des liens de complicité et de confiance, mais également des controverses et des conflits dans la réalisation de ces projets de traduction. Enfin, et surtout, elle offrirait la possibilité d'envisager la traduction, par-delà les oppositions source/cible, comme une activité s'inscrivant dans une logique de réseaux. Il s'agirait dès lors de mieux comprendre la nature de ces réseaux et comment ces derniers structurent et sont en même tant structurés par le processus de traduction. Bref, cette approche, en évitant le biais « individualisation » et « rationalisation » de l'objet d'étude - que génèrent les méthodes d'analyse traditionnelles, serait susceptible d'enrichir, sur un mode empirique, les recherches descriptives actuelles et d'approfondir, à terme, les réflexions et tentatives de reconceptualisation amorcées.

\section{Conclusion}

Cet article a exploré les liens entre la traductologie et l'ethnographie sous l'angle de la production des connaissances. Après avoir rappelé la nature et les implications des constats effectués par les traductologues postcoloniaux, on s'est tourné vers l'anthropologie afin d'y rechercher des éléments de réponse à la question suivante, qui est au cour des théories postcoloniales: who translates? Si l'ethnographie et la traduction sont des pratiques en partie distinctes, la réflexion des anthropologues sur la construction des connaissances permet de souligner un aspect qui vaut également pour la traduction interlinguistique, mais qui est relativement peu reconnu et peu étudié par les traductologues. Elle nous invite, entre autres, à penser la traduction d'un point de vue dynamique, à l'aborder comme un processus de production collectif, donc social, un travail qui passe par une confrontation de points de vue. Dès lors, le produit fini, la traduction-texte, n'apparaît plus comme le «simple» reflet des normes d'une société donnée ou de la subjectivité d'un traducteur, mais bien plutôt comme le résultat des relations qui se sont tissées entre les actants (y compris les machines) qui ont participé à sa réalisation. Cette conclusion jette un nouvel éclairage sur le débat éthique et ouvre de nouvelles avenues de recherche pour le paradigme descriptif. Les paragraphes suivants développent ces deux aspects.

Comme le souligne Gillian Lane-Mercier, en réponse à l'idéalisme d'Antoine Berman, «ce n'est qu'en donnant droit de cité à l'étranger du dedans qu'il est possible d'ouvrir une brèche à partir de laquelle une appréhension de l'étranger du dehors - aussi médiatisée soit-elle sur les plans culturel et institutionnel - peut être envisagée» (1998: 84). En ce sens, le foreignizing proposé par Venuti offre une option intéressante. Cela dit, outre le fait qu'elle ne se prête pas à tous les textes et contextes, cette éthique consistant à "marquer» la différence, à introduire des "minority constituants», contribue malgré tout, jusqu'à un certain point, à perpétuer la marginalisation des éléments en question. Le premier défi de la traduction (dans une perspective postcoloniale) ne serait-il pas plutôt d'aborder ces éléments en eux-mêmes, cesser d'y voir des «minority elements»? Bien plus qu'un choix entre l'une ou l'autre stratégie d'écriture, la traduction en contexte postcolonial exige, avant toute chose (avant de les substituer par d'autres) que l'on replace les «minority elements» du texte de départ au cour du processus interprétatif, qu'on lise ce texte, non pas uniquement, mais avant tout, selon leur point de vue. Or, les éléments en question étant précisément peu reconnus et, lorsqu'il s'agit de langues, peu standardisés, acquis plutôt qu'appris, 
une telle approche revient à encourager l'élaboration de projets de traduction collectifs, voire même à contredire le vieux poncif selon lequel on traduit toujours mieux dans sa langue maternelle et donner, plutôt, aux «lecteurs de départ» pour lesquels ces «elements» sont beaucoup plus que des «minority elements» le contrôle du processus de traduction. Parce qu'il cherche à favoriser l'hétérogénéité dans le contexte d'arrivée, le foreignizing perpétue le paradigme traditionnel, celui de la traduction vers le familier. Il maintient aussi le culte du texte. Au contraire, rapatriée dans le champ de la traductologie, la réflexion des anthropologues - du moins ceux dont on s'est inspirée - permet tout d'abord de penser l'éthique en des termes qui ne soient pas exclusivement formels mais qui tiennent compte, plutôt, de l'ensemble du processus et, par conséquent, du rôle, de la position et des relations entre les actants susceptibles d'y participer. Dès lors, on peut se demander si l'étude de ces interactions ne devrait même précéder toute tentative visant à définir une éthique de la traduction. J'en viens donc au second point: l'apport de la réflexion ethnographique sur le plan théorique.

Selon Michael Cronin, les sociologues et anthropologues, y compris ceux qui s'intéressent aux phénomènes de mondialisation, ont manifesté peu d'intérêt pour les problématiques linguistiques et traductologiques. Cette lacune a été également relevée, dans le cas de la sociologie, par Simeoni (2002) qui, au-delà de la critique, en a expliqué les raisons institutionnelles et politiques. Sturge (1997: 1998) reproche à Clifford Geertz ainsi qu'à l'auteur de Routes: Travel and Translation in the Late Twentieth Century, James Clifford, de n'accorder que bien peu d'importance à la traduction interlinguistique. Quant aux travaux de Bruno Latour, ils n'ont encore eu aucun écho chez les traductologues, y compris ceux qui s'intéressent à la traduction dans une perspective sociologique. De façon générale, on reproche donc à ces anthropologues d'avoir envisagé la traduction sous un angle purement métaphorique, de ne pas s'être intéressés aux phénomènes de traduction interlinguistique. La critique est justifiée, mais également surprenante de la part de traductologues post-structuralistes dont les travaux tendent précisément à élargir leur champ de recherche au-delà des phénomènes de transfert linguistique, si ce n'est à briser la distinction traditionnelle entre traduction interlinguistique, intralinguistique et intersémiotique. On peut effectivement regretter que les anthropologues ne se penchent pas un peu plus sur leurs propres pratiques de traduction (au sens strict) et les encourager à le faire, ou bien le faire à leur place, ce qu'a d'ailleurs entrepris Kate Sturge (1997). Au terme de cette réflexion, on peut toutefois se demander si la traductologie n'aurait pas aussi intérêt à se «réapproprier» le concept de traduction défini par les anthropologues tels que Bruno Latour. Ce dernier n'a pas fait de la traduction interlinguistique son premier objet de recherche. Par contre son approche, fondée sur des présupposés théoriques identiques à ceux qui se dessinent aujourd'hui en traductologie, permet tout d'abord de désamorcer le clivage épistémologique dont la discipline demeure prisonnière et offre une ligne méthodologique solide à partir de laquelle l'étude empirique des processus de traduction/production, conduite dans le respect des partis pris théoriques d'hybridité et de dialectisme, demeure possible et valable. Une telle étude reste à faire. Les modalités de sa réalisation feront l'objet d'un prochain article. 


\section{NOTES}

* L’auteur tient à remercier Jean-Sébastien Marcoux et Daniel Simeoni pour leur lecture et les commentaires qu'ils ont apportés aux versions préliminaires de cet article dont la dernière partie est inspirée d'une proposition de recherche à laquelle ils ont activement participé.

1. Les liens entre la traduction et l'écriture postcoloniale ont également été abordés, entre autres, par Prasad (1999) pour l'Inde, Bandia (1994, 1996) pour l'Afrique, ainsi que D'Costa (1984), Akai (1997) et Confiant (2000) pour la Caraïbe.

2. Les stratégies d'écriture ethnographique «traditionnelles» ont également fait l'objet d'analyses (Asad 1979; Clifford 1980; Clifford et Marcus 1986; Sturge 1997). Pour une critique des nouveaux modes d'écriture proposés, entre autres, par Clifford et Marcus (1986), voir Said (1989) et Mascia Lees et al. (1989).

3. Dans un article publié en 1972 Nash et Wintrob mentionnent quatre éléments explicatifs: 1) le resserrement des liens entre les ethnographes et leurs informateurs, resserrement encouragé par une certaine idéalisation du «terrain» reposant sur le principe d'immersion et la valorisation de la participation sur l'observation; 2) la démocratisation de la profession instaurant une plus grande compétition entre les chercheurs; 3 ) la production de plusieurs études contradictoires d'un même contexte, mettant en doute la validité des données recueillies et des études en question; 4) l'accession à l'indépendance des pays colonisés. Au vu des commentaires de dix pages figurant à la suite de cet article, de même que d'autres articles ayant abordé cette question (Hymes (ed.) 1972, Scholte 1971), les anthropologues n'accordent pas tous le même poids aux quatre facteurs, certains en relevant d'ailleurs d'autres, comme, par exemple, la concurrence des autres champs de recherche, l'influence de la psychologie cognitive, le passage d'une anthropologie fonctionnaliste à une anthropologie sémantique. Cela dit, de façon générale, le processus de décolonisation est le facteur qui revient, de façon récurrente, dans toutes les analyses.

4. Voir Laplantine (1987: 137-144): «L'anthropologie dynamique».

5. Voir entre autres les recherches de Callon et al. (1986), Latour et Woolgar (1988), Callon (1989), Knorr-Cetina (1981, 1999), Knorr-Cetina et Mulkay (1983), Löwy (1996).

6. Christine Raguet-Bouvart (ed.) Palimpsestes, 12.

7. Dans une perspective historique, Simeoni (1998) souligne à juste titre la nécessité et la difficulté d'accéder à la trajectoire des traducteurs, difficulté liée non seulement à l'invisibilité légendaire de ces derniers mais au fait que leurs textes (lorsqu'ils sont encore disponibles) ont été, plus que tout autres, sujets à modifications, falsifications, altérations «with little hope for the analyst to disentangle the various hands, minds and hearts responsible for the final product» (Simeoni 1998: 32). L'importance de ces intermédaires, et donc la difficulté de saisir et plus encore de définir le "sujet traduisant», est également mentionnée par Robinson (2001).

\section{RÉFÉRENCES}

AKAI, J. (1997) : «Creole... English: West Indian Writing as Translation», TTR 10-1, p. 165-196.

Asad, T. (ed.) (1973): Anthropology and the Colonial Encounter, Atlantic Highlands, Humanities Press International.

Asad, T. (1979): «Anthropology and the Analysis of Ideology», Man 14-4, p. 607-627.

BANDIA, P. (1994): «On Translating Pidgins and Creoles in African Literature», TTR 7-2, p. 93-114.

BAndia, P. (1996) : «Code-Switching and Code-Mixing in African Creative Writing: Some Insights for Translation Studies», TTR 9-1, p. 139-154.

Bassnett, S. (1998): "The Translation Turn in Cultural Studies», in Constructing Cultures, Lefevere, A. and S. Bassnett (eds.), Clevedon, Multilingual Matters, p. 123-140.

Bassnett, S. and H. Trivedi (1999): Postcolonial Translation, Theory and Practice, London and New York, Routledge.

Benjamin, W. (1997) : «L'abandon du traducteur», traduction par Alexis Nouss et Laurent Lamy TTR, 10-2, p. 13-70.

Berman, A. (1984): L'épreuve de l'étranger, Paris, Gallimard.

Berreman, G. D. (1968) : «Is Anthropology Alive? Social Responsibility in Social Anthropology», Current Anthropology 9-5, p. 391-396.

Buzelin, H. (2004): Sur le terrain de la traduction, Toronto, Gref (à paraître). 
Callon, M., John Law, Arie Rip (1986): Mapping the Dynamics of Science and Technology, London, McMillan.

Callon, M. (1989): La science et ses réseaux, Paris, La Découverte.

Chapdelaine, A. et G. Lane-Mercier (dirs.) (2001) : Faulkner: Une expérience de retraduction, Montréal, Presses de l'Université de Montréal.

Clifford, J. (1980) : «Fieldwork, Reciprocity, and the Making of Ethnographic Texts: The Example of Maurice Leenhardt", Man 15-3, p. 518-532.

Clifford, J. (1997): Routes: Travel and Translation in the Late Twentieth Century, Cambridge (Mass) and London (England), Harvard University Press.

Clifford, J. and G. E. Marcus (eds.) (1986): Writing Culture: The Poetics and Politics of Ethnography, Berkeley and London, University of California Press.

Confiant, R. (2000) : «Traduire la littérature en situation de diglossie», Palimpsestes, 12, p. 49-60.

Crick, M. (1982) : «Anthropological Field Research, Meaning Creation and Knowledge Construction ", in Semantic Anthropology, Parkin (ed.), ASA monograph 22, London, Academic Press, p. 15-38.

Cronin, M. (2000): Across the Lines: Travel, Language, Translation, Cork, Cork University Press.

Cronin, M. (2003): Translation and Globalization, London and New York, Routledge.

Davies, C. A. (1999): Reflexive Ethnography, London and New York, Routledge.

D'Costa, J. (1984): «Expression and Communication: Literary Challenges to the Caribbean Polydialectal Writers. » Journal of Commonwealth Literature, 19-1, p. 123-141.

Douglas, M. (1995): «Forgotten Knowledge», in Shifting Contexts, Marilyn Strathern (ed.), London and New York, Routledge, p. 13-27.

Fabian, J. (1971): «On Professional Ethics and Epistemological Foundations», Current Anthropology 12-2, p. 230-232.

Fabian, J. (1983): Time and the Other: How Anthropology Makes Its Object, New York, Columbia University Press.

Folkart, B. (1991): Le conflit des énonciations: traduction et discours rapporté, Québec, Les Éditions Balzac, «l'Univers des discours».

Gough, K. (1968) : «Anthropology and Imperialism», Monthly Review 19-11, p. 12-27.

Hatfield, C. R. (1975): «Fieldwork: Towards a Model of Mutual Exploitation» in Cultural and Social Anthropology» Introductory Readings in Ethnology, Hammond (ed.), New York, Mcmillan.

Heilbron, J. (1999) : «Towards a Sociology of Translation », European Journal of Social Theory 24, p. 429-444.

House, J. (2001) : «Translation Quality Assessment: Linguistic Description versus Social Evaluation », META 46-2, p. 243-257.

Hymes, D. (ed.) (1999 [@1972]): Reinventing Anthropology, New York, Pantheon Books.

JACQuemont, R. (1992): "Translation and Cultural Hegemony: The Case of French-Arabic Translation", in Rethinking Translation, Lawrence Venuti (ed.), London and New York, Routledge, p. 139-158.

Jarvie, I. C. (1969): «The Problem of Ethical Integrity in Participant Observation», Current Anthropology 10-5, p. 505-508.

Jongmans, D. R. and P. C. W. Gutkind (eds.) (1967): Anthropologists in the Field, Assen, Van Gorcum.

Jorgensen, J. G. (1971) : «On Ethics and Anthropology», Current Anthropology 12-3, p. 321-334.

Kadish, D. Y. and F. Massardier-Kenney (eds) (1994): Gender and Race in French Women's Writing, 1783-1823, Kent (Ohio), The Kent State University Press.

Knorr-Cetina, K. D. (1981): The Manufacture of Knowledge: An Essay on the Constructivist and Contextual Nature of Science, Oxford, Pergamon Press.

Knorr-Cetina, K. D. (1999): Epistemic Cultures: How the Sciences Make Knowledge, Cambridge, Mass., Harvard University Press.

Knorr-Cetina, K. D. and M. Mulkay (eds.) (1983): Science Observed: New Perspectives on the Social Study of Science, London: Sage Publ. Co. 
Lane-Mercier, G. (1998): «Le travail sur la lettre: politique de décentrement ou tactique de réappropriation", TTR 11-1 p. 65-88.

Lane-Mercier, G. (2001) : «L'impossible unicité: le conflit des subjectivités et des réceptions » in Faulkner: Une expérience de retraduction, Chapdelaine et Lane Mercier (dirs.), Montréal, Presses de l'Université de Montréal, p. 131-178.

Laplantine, F. (1987): L'anthropologie, Paris, Seghers.

Latour, B. et S. Woolgar (1996): La vie de laboratoire: la production des faits scientifiques, Paris, La découverte.

Latour, B. (1989): La science en action, traduction de l'anglais par Michel Biezunski, Paris, La découverte.

Latour, B. (1997): Nous n'avons jamais été modernes: Essai d’anthropologie symétrique, Paris, La découverte.

Leach, E. R. (1968): Critique de l'anthropologie, Paris, PUF.

Leclerc, G. (1972): Anthropologie et colonialisme, Paris, Fayard.

Leiris, M. (1950) «L'ethnographe devant le colonialisme", Les temps modernes 6-58, p. 357-374.

Levine, S. J. (1991): The Subversive Scribe, Translating Latin American Fiction, Saint Paul (Minn.), Graywolf Press.

Lewis, R. A. (2002): "Non-Standard Languages and Translation», Journal of Contemporary Thought, p. 25-36.

LI, D. (2000): «Tailoring Translation Programs to Social Needs», Target 12-1, p. 127-149.

Löwy, I. (1996): Between Bench and Bedside: Science, Healing, and Interleukin-2 in a Cancer Ward, Cambridge, Mass., Harvard University Press.

Lundberg, C. (1968): «A Transitional Conception of Fieldwork», Human Organization 27, p. 45-49.

Maquet, Jacques J. (1964): «Objectivity in Anthropology», Current Anthropology 5-1, p. 47-55.

Mascia-Lees, F. et al. (1989): "The Postmodernist Turn in Anthropology: Cautions from a Feminist Perspective», Signs 15-1, p. 7-33.

Mounin, G. (1963): Les problèmes théoriques de la traduction, Paris, Gallimard.

Nash, D. and R. Wintrob (1972): «The Emergence of Self-Consciousness in Ethnography», Current Anthropology 13-5, p. 527-542.

NidA, E. (1945): «Linguistics and ethnology in Translation problems», Word 2, p. 194-208.

Nouss, A. et F. Laplantine (1997): Le métissage: un exposé pour comprendre, un essai pour réfléchir, Paris, Flammarion, Collection «Dominos».

PÁlsson, G. (ed.) (1993): Beyond Boundaries: Understanding, Translation and Anthropological Discourse, Oxford and Providence, Berg.

Polezzi, L. (2001): Translating Travel: Contemporary Italian Writing in English Translation, Aldershot, Ashgate.

Prasad, G.J.V (1999) : «Writing Translation: The Strange Case of the Indian English Novel», in Postcolonial Translation: Theory and Pratice, Bassnett and Trivedi (ed.), London and New York, Routledge, p. 41-57.

Raguet-Bouvart, C. (ed.) (2000) : «Traduire la littérature des Caraïbes», Palimpsestes 12.

Robinson, D. (1997): Translation and Empire, Manchester, St-Jerome.

Robinson, D. (2001): Who Translates? Translator Subjectivities beyond Reason, Albany, State University of New York Press.

SAID, E. (1989): «Representing the Colonized: Anthropology's Interlocutors.» Critical Inquiry, 15, p. 205-225.

Scholte, B. (1971): «Discontents in Anthropology», Social Research 38-4, p. 777-807.

SÉGuINot, C. (1995): «Translation and Advertising: Going Global», in Cultural functions of translation, Schäffner and Kelly-Holmes (eds.), Clevedon, Multilingual Matters, p. 55-71.

Sela-Sheffy, R. (2000): "The Suspended Potential of Culture Research in TS », Target 12-2, p. 345-355.

Simeoni, D. (1995): «Translating and Studying Translation: The view from the Agent», META 40-3, p. 445-460. 
Simeoni, D. (1998) : «The Pivotal Status of the Translator's Habitus.» Target, 10-1, p. 1-39.

Simeoni, D. (2002): "Translation and Society: The Emergence of a Conceptual Relationship ", Journal of Contemporary Thought, p. 9-24.

Simon, S. (1999): «Translating and Interlingual Creation in the Contact Zone: Border Writing in Quebec ", in Postcolonial Translation, Theory and Practice, Bassnett and Trivedi (eds.), London and New York, Routledge, p. 58-74.

Simon, S. (1996): Gender in Translation, London and New-York, Routledge.

Simon, S. and P. St-Pierre (eds.) (2000): Changing the Terms, Ottawa, University of Ottawa Press.

SpIVAK, G. C. (2000 [๔ 1993]): "The Politics of Translation», in The Translation Studies Reader, Venuti (ed.), London and New York, Routledge, p. 397-416.

Sturge, K. (1997): "Translation Strategies in Ethnography», The Translator 3-1, p. 21-38.

Sturge, K. (1998): «James Clifford: Routes. Travel and Translation in the Late Twentieth Century», The Translator 4-2, p. 375-379.

Toury, G. (1980): In Search of a Theory of Translation, Tel Aviv, The Porter Institute.

Tүмосzко, M. (1999): Translation in a Postcolonial Context, Manchester, St-Jerome.

Түмосzко, М. (1999b) : «Postcolonial writing and literary translation», in Postcolonial Translation, Theory and Practice, Bassnett and Trivedi (eds.), London and New-York, Routledge, p. 19-40.

Tyмосzкo, M. (2000) : «Translations of Themselves: The Contours of Postcolonial Fiction », in Changing the Terms, Simon and St-Pierre, Ottawa, Presses de l'Université d'Ottawa, p. 147-163.

Valero-Garcès, C. (1995): "Modes of Translating Culture: Ethnography and Translation», META 40-4, p. 556-563.

Venuti, L. (1998): The Scandals of Translation. Towards an Ethics of Difference, London and New York, Routledge.

Venuti, L. (1996) : «Translation, Heterogeneity, Linguistics», TTR 9-1, p. 91-116.

Wolf, E. (1964): Anthropology, Englewood Cliffs, Prentice-Hall.

Wolf, M. (2000): "The Third Space in Postcolonial Representation», in Changing the Terms, Simon and St-Pierre (eds.), Ottawa, Presses de l'Université d'Ottawa, p. 127-143. 